\title{
La comunicación corporativa a través de las páginas Web: el caso de las universidades españolas
}

\section{Corporate Communication in Web Sites: the Case of Spanish Universities}

Alejandro Tapia Frade. Universidad Europea Miguel de Cervantes

Begoña Gómez Nieto. Universidad Europea Miguel de Cervantes

Recibido: 25-II-2011 - Aceptado: 14-VII-2011

Resumen:

La comunicación corporativa supone un activo de suma importancia para cualquier organización. También para las universidades, que se encuentran en un proceso de convergencia que exige la generación de un discurso corporativo que se adapte al nuevo entorno competidor. Así, este trabajo analiza la comunicación corporativa de las universidades españolas a través de sus portales Web mediante la técnica de análisis de contenido con perspectiva cuantitativa. Los resultados ponen de manifiesto que aunque la presencia de una sección específica destinada a ésta en las páginas analizadas es mayoritaria, la gestión de contenidos de la misma no puede calificarse de buena, ya que no se incluye toda la información que debería, y todavía más, no se aprovecha la flexibilidad del soporte para generar discurso corporativo multimedia.

Palabras clave:

Comunicación corporativa, Internet, visibilidad, portal Web, transparencia, universidad.

Abstract:

Corporate communication is a paramount strategic asset for any organization. Also for the universities, which are in a European convergence process that requires communication efforts targeting the generation of a corporate speech to suit the new competitor enviroment. Therefore, this paper analyzes corporate communications in Spanish universities through their Web portals. This was done using the technique of content analysis. The results highlight the inadequate management of corporate communication on the Internet. Although the presence of a specific section in the pages analyzed the majority, content management cannot be regarded as good, since it does not contain all information that should, and even more, is not utilized properly support flexibility for generating multimedia corporate speech.

Keywords:

Corporate Communication, Internet, Visibility, Web Portal, Transparency, University. 


\section{Marco teórico}

La imagen corporativa supone un activo fundamental para las organizaciones, creando valor y estableciéndose como un activo intangible estratégico de primer nivel para la misma, ocupando espacio en la mente de sus públicos, facilitando su diferenciación respecto a organizaciones competidoras, y disminuyendo la influencia de potenciales factores de tipo situacional en el proceso de decisión de compra (Capriotti, 2005:10-11). Esta imagen corporativa es descrita por Van Riel (2000: 27) como el retrato que se tiene de una empresa, cómo ésta es percibida por los públicos objetivo.

La imagen de una empresa ha de considerarse como "el resultado interactivo que un amplio conjunto de comportamientos de aquella producen en la mente de sus públicos”, lo que le lleva a considerar la imagen como el resultado conjunto integrado de la identidad de la empresa y del trabajo corporativo, entendido como "todas aquellas posibles actuaciones, voluntarias o involuntarias, con intención comunicativa o sin ella, que hacen identificar en la mente de los públicos la imagen y la identidad de la empresa” (Sanz de la Tajada, 1996: 21).

Pero una imagen de empresa no es algo absoluto sino que, en general, se diferencia sensiblemente según los públicos. Así, por ejemplo, los proveedores de una empresa pueden tener una imagen muy diferente de la que poseen sus clientes y de la de los propios empleados y/o accionistas. De ahí la importancia de la diferenciación de los públicos de la empresa. Para el establecimiento de una adecuada estrategia de comunicación al servicio de los objetivos propuestos, es fundamental llegar a una precisa definición de los diferentes tipos de destinatarios de las acciones (segmentos o población objetivo). La relación de la empresa con sus públicos no se produce exclusivamente con cada uno de ellos, sino que existe un entramado de relaciones de influencia, lo que dota a esta definición de públicos de cierta complejidad (Sanz de la Tajada, 1996: 22).

En el caso de las universidades, también existe una diferenciación de públicos: el principal, serían los clientes que utilizan la entidad para su formación (alumnos de grado, licenciatura, master, doctorado, profesores visitantes, usuarios de centros de investigación asociados a universidades, etc.). Pero también son públicos los medios de comunicación, puesto que son generadores de opinión pública y la sociedad en general. Así los mensajes serán diferentes atendiendo a esta distinción.

La identidad corporativa denota la suma total de todas las formas de expresión que una empresa utiliza para ofrecer una perspectiva de su naturaleza. Es la forma en la que una empresa se presenta al público objetivo mediante el uso de símbolos, comunicación y comportamientos. Estos tres elementos constituyen el llamado mix de identidad corporativa (Van Riel, 2000: 29). 
El material de trabajo de la función de comunicación es la estrategia de la compañía, incluyendo su identidad, la imagen que desea proyectar y lo que debería hacer para conservar esa imagen. El papel de la comunicación es -o debería ser- una función estratégica dentro de la dirección de la empresa, basada en el qué de la compañía. Si la identidad de una compañía es "lo que realmente es”, y su imagen es lo que otros piensan o creen que es, la tarea de la comunicación será relativamente fácil: unir ambos conceptos. Pero los conceptos de "identidad" e "imagen" son complejos, tanto si se refieren a personas como a empresas u organizaciones.

En el caso de las universidades, la página Web se conforma como un vehículo transmisor de la imagen de la institución de máxima relevancia, puesto que es uno de canales de información más utilizados en la búsqueda de información, de ahí la necesidad de estructurar y diseñar de forma atractiva y eficaz la misma.

Dicha imagen se configura a partir de la identidad de la empresa, que tiene dos dimensiones complementarias: la identidad visual o sígnica, que tiene que ver con la forma física de la identidad empresarial, y la dimensión conceptual (Sanz de la Tajada, 1996:32), que se relaciona con el comportamiento, la cultura y la personalidad corporativa (Villafañe, 1993: 28-29).

Actualmente la imagen corporativa de las empresas en Internet se ha consolidado como un factor estratégico clave en el ámbito empresarial, como demuestra la amplia labor investigadora que se está desarrollando sobre este tema.

Su grado de importancia es especialmente elevado en la circunstancia actual de las universidades, habida cuenta de su intenso proceso de adaptación al Espacio Europeo de Educación Superior. Dicho proceso, además de la mera reorganización de planes de estudio y métodos de enseñanza, exige un importante esfuerzo comunicativo de la propuesta corporativa específica de cada universidad a dicho espacio ya que el contexto competidor se ha visto seriamente ampliado para los públicos fundamentales que son objeto de su actividad, profesores, alumnos de grado, licenciatura, máster, doctorado, profesores visitantes, usuarios de centros de investigación asociados a universidades, etc.

Internet supone sin duda un amplio escaparate en el que promover tal imagen. Su ya amplia cobertura, estimado en el 50,5\% de la población española (EGM, 2009: 2), hace sugerir la importancia de este medio en la difusión de información en general, siendo por tanto un medio vehicular apto para la gestión de información corporativa, como se ha demostrado (Herranz de la Casa, 2010: 316; Martínez Grás, 1998) ${ }^{1}$ Todavía

En concreto se ha señalado que “El elevado volumen de información junto a la necesidad de una gestión global de la comunicación 
más, la evolución de Internet hacia la llamada Web participativa, o Web 2.0 (Fumero, A., Roca, G. y Sáez Vacas, F., 2007), tiene entre otras como característica esencial el paso de un perfil de usuario como simple consumidor de información a otro de usuario activo, creador de información, (Freire, 2007:83) lo que hace sugerir la posibilidad de uso de este canal por parte de las universidades como constructores de discurso corporativo a través de la interacción con sus públicos.

En una primera aproximación sobre el tema, en un estudio se analizaron 17 sitios Web (uno por comunidad autónoma) revisando indicadores de contacto, legibilidad y ergonomía, navegación y representación, recuperabilidad, velocidad de descarga y luminosidad, con conclusiones poco alentadoras (Gil Leiva y Moya Martínez, 2001).

Un poco después se comprobó que el tamaño de las webs universitarias españolas y su grado de interconexión en relación a sus homónimas europeas era el adecuado (Thelwall y Aguillo, 2003: 291-305), si bien otro estudio ese mismo año puso de manifiesto que su nivel de accesibilidad no lo era (Térmens Graells, M., Ribera Turró, M. y Sulé Duesa, A., 2003: 21). Otro estudio posterior similar incidió en la diferencia existente entre las webs estadounidenses y europeas (especialmente francesas, alemanas e inglesas) respecto a su tamaño y visibilidad (Aguillo y Granadino, 2006: 73).

También se han destacado en un análisis de una muestra de webs universitarias varias deficiencias, entre ellas la pobre presencia del idioma inglés en las mismas (Pinto Molina, 2004: 345-370), que como se muestra en este trabajo, ha sido ampliamente subsanado.

La visibilidad de las webs universitarias ha sido un tema también tratado. Así, se puso de manifiesto en un estudio sobre webs universitarias públicas y privadas de España una visibilidad ligeramente superior de las universidades públicas sobre las privadas, que se explica en parte por el diferente tamaño general de estas universidades. También concluyó que la barra de navegación es un elemento más frecuente en universidades públicas que privadas, y que este elemento suele situarse en la parte superior derecha de la página Web (46\% de veces en universidades públicas, frente a 17\% en organizaciones privadas) (Martínez Méndez, 2008: 87-106).

Fuera de España, en un análisis sobre websites universitarias americanas, se determinó que las universidades más liberales favorecían la comunicación con los estudiantes a través de las páginas Web (Gordon y Berhow, 2009: 150-152). En otro estudio, en este caso francés se señaló en un análisis cierta similitud de ca-

tanto entre colectivos como para la comunidad universitaria y la sociedad en general, convierten a Internet en uno de los canales de comunicación más aptos para la gestión de la información que se produce en cada una de las dependencias de nuestra universidad" 
tegorías en las páginas de inicio de las webs universitarias, que impedían la particularización de la propuesta corporativa de las mismas (Reymond, 2006: 414 -419).

Otro estudio ha mencionado que es Europa la región que mayor preocupación muestra por la Responsabilidad Social Empresarial (RSE) (Cruz, Rodríguez y Abreu, 2010: 13), justificado sin duda por el creciente interés de ciudadanos, clientes y consumidores, que presionan a las organizaciones y convierte, al menos en parte, la responsabilidad social en un activo comercial estratégico en el entorno empresarial (García, Iturrioz, Mateu y Palomo, 2011: 122; Aguadero, 2010: 69).

Finalmente, Castañeda, Ruiz, Viloria, Castañeda y Quevedo (2007) proponen en una interesante reflexión mayor pluridisciplinariedad del conocimiento universitario, al situar la responsabilidad social de la universidad al frente de la solución de problemas complejos donde no cabe la dislocación del saber.

La disparidad de resultados constata el fuerte cambio que ha sufrido la comunicación corporativa en los últimos años debido al desarrollo de Internet, ha impulsado la realización de este trabajo. También ha de considerarse si la universidad es de titularidad pública o privada, ya que la primera tiene exclusivamente como objetivo el servicio a la sociedad, y la segunda constituye en muchos casos un negocio. Esta diferencia implica también un aumento sensible de coste para el alumnado, que podría justificar en el caso de las universidades privadas el uso de un discurso corporativo más intenso, agresivo, o el uso más frecuente de formatos multimedia. Por ello, consideramos posible la existencia de diferencias relevantes en cuanto a contenidos dependiendo de si la universidad es pública o privada.

En definitiva, este trabajo se centra en la exposición de información corporativa de las universidades públicas y privadas españolas en sus páginas Web, con el objetivo de determinar si dicha comunicación está adecuadamente estructurada y los contenidos son los acertados en ambos casos.

\section{Objetivos e hipótesis de partida}

Por cuanto antecede, el objetivo principal de este trabajo es el análisis de la situación comunicativa corporativa de las páginas Web universitarias públicas y privadas españolas, que tiene dos dimensiones diferenciadas: el contenido, es decir, qué elementos corporativos se incluyen en las páginas Web, y la estructura, es decir, en qué lugar de la página Web se ubican y si en efecto existe una sección específica destinada a dicho tipo de información.

De los mencionados objetivos, señalamos las siguientes hipótesis de partida: 
H1) Las páginas Web de las universidades públicas y privadas albergan, en su mayoría, una sección específica destinada a la comunicación corporativa.

H2) Las páginas Web de las universidades públicas no contienen, al menos en su mayoría, información concerniente a comunicación corporativa, al menos biografías, informes financieros, historia, valores y misión, organigrama, calendario de eventos, apartado de RSC y logotipos o símbolos. Las universidades privadas, al contrario, sí contienen dicha información.

H3) Las páginas Web de las universidades públicas no utilizan formatos multimedia tales como vídeo en la generación de discurso corporativo. Las universidades privadas, en sentido opuesto, si utilizan dicho formato.

H4) Las páginas Web de las universidades privadas usan en mayor medida herramientas propias de la Web 2.0, tales como blogs, servicios de alerta por móvil, servicios de sindicación de noticias por RSS, presencia en redes sociales y canales de YouTube, que las universidades de titularidad pública.

\section{Metodología}

A fin de conocer en qué situación se encuentran las universidades españolas en lo relativo a la comunicación corporativa por Internet, se decidió el uso de la técnica del análisis de contenido con perspectiva cuantitativa. Para ello se realizó una plantilla de análisis² compuesta por 43 elementos que estructuran e ilustran las posibilidades comunicativas que ofrecen dichas organizaciones en cuanto a comunicación corporativa en Internet.

Se determinó revisar la presencia o ausencia de una sección específica destinada a comunicación corporativa y en su caso en qué nivel estructural se encontraba -medido a través del conteo de clicks necesarios para acceder a dicha sección. Dentro de este capítulo de información corporativa se examinó si se proporcionaba información de la historia de la institución -y dentro de ésta, si existen referencias explícitas a mitos- , de biografías -y en su caso cuáles- , valores y misión de la organización, organigrama del mismo, calendarios de eventos, informes financieros, información sobre responsabilidad social corporativa, elementos de identidad corporativa, etc.

También se valoró si en la comunicación se utilizaba únicamente texto o si se aprovechaban las cualidades del medio y se incluía fotografía, audio y vídeo.

2 Dicha plantilla de análisis, que puede verse en el anexo, tiene precedentes en otros estudios de similar propósito. (Tapia, A. et al., 2009). 
En lo relativo a la Web 2.0, también llamada la Web participativa, se analizó, al igual que en caso anterior, la presencia y ubicación de elementos que potencialmente podrían ser de utilidad para la generación de discurso corporativa como los blogs, los servicios de alerta por móvil y los servicios de sindicación de noticias por RSS.

Se usó la misma escala para determinar no sólo presencia o ausencia del elemento, sino también el grado de acierto de tal hipotética presencia en una sección concreta-la de información corporativa- en todos los casos. Así, dicha escala constaba de cuatro elementos que transitaban entre la presencia en el lugar más acertado a dicho contenido ("Está, en la sección de información corporativa") a la ausencia del mismo ("No está"), pasando por dos estadios intermedios: "Está, pero en sección de sala de prensa" (menos acertado a nuestro parecer que la sección de información corporativa, pero más acertado que en otra sección distinta de ambas) y "Está, pero fuera de ambas secciones" (la información corporativa y la de sala de prensa).

Finalmente, también se analizó la presencia de las universidades en redes sociales y la existencia de canales de YouTube, si bien no se concretó en qué parte de la página Web se encontraban, ya que dichas herramientas bien podrían tener un propósito que excediera la generación de discurso corporativo y fueran utilizadas como herramientas de relación genéricas con sus públicos. En cualquier caso, la presencia de las mismas es fundamental ya que posibilita la generación de discurso corporativo a través de un medio muy usado por un público fundamental para las universidades, los estudiantes.

También se revisó si la presencia en el lugar correcto de cierto elemento hacía más probable la presencia de otros, es decir, si existía alguna tendencia a la agrupación de los elementos estudiados en lugares concretos de la Web. Para ello, se realizaron correlaciones usando los estadísticos Rho de Spearman y Tau_b de Kendall.

Finalmente, también se realizó un análisis de fiabilidad de dicha escala mediante el estadístico Alfa de Cronbach, con un resultado global de 0,531, que aún siendo bajo, resulta válido si el propósito es únicamente la investigación.

Se ejecutó dicha plantilla de análisis sobre los sitios Web de las 74 universidades de titularidad pública y privada en España ${ }^{3}$. La recogida de información se efectuó durante el mes de diciembre de 2010.

Por tanto, nos encontramos ante el análisis de la totalidad del universo de estudio, o lo que es lo mismo, un estudio por censo. Dicho estudio fue posible dado el limitado tamaño y la amplia disponibilidad del uni-

El listado de universidades analizadas puede apreciarse en la Tabla I. 
verso, que permitió la recogida de información en un corto espacio de tiempo, consideración ésta imprescindible ante un estudio de tipo transversal como el que nos ocupa.

Los resultados se estratificaron según la titularidad de la universidad, ya que tal y como se mencionó antes, entendemos que la misma puede ser objeto de diferencias relevantes debido al diferente objetivo inherente a cada tipo de ellas: las privadas tienen en su mayoría ánimo de lucro, son en sí mismo un negocio, y las públicas no tienen tal fin, sino únicamente el servicio a la sociedad. Por tanto, si los objetivos son distintos, el discurso corporativo puede también ser objeto de diferencias relevantes. Para la explotación de datos se utilizó el software específico SPSS v.15.

\section{Resultados: La comunicación corporativa en página Web}

\subsection{Visibilidad en la Web}

La primera consideración acerca de la comunicación en Internet entendemos que debe plantearse en términos dicotómicos, es decir, considerar si efectivamente estas organizaciones tienen o no página Web. Los resultados, tal y como puede apreciarse en la tabla posterior, no dejan lugar a dudas: todas las universidades de España, sean públicas o privadas, exponen información corporativa en este medio, lo que implica que al menos poseen el soporte necesario para transmitir información en el medio.

Otra cuestión es el alcance que se desee tener en este medio. Así, la primera consideración de contenido debe ser en qué idioma o idiomas está disponible dicha página. No obstante, la elección dependerá de forma notoria del público al que se desea alcanzar. En el caso de las universidades, la elección mayoritaria pasa por el empleo de más de dos idiomas (usualmente español, la lengua cooficial e inglés), reflejando no sólo cierta vocación internacional, sino también acusando cierta tendencia regional, si bien cumpliendo en muchos casos la normativa legal vigente. Los resultados pueden apreciarse en la tabla II.

Una de las primeras consideraciones a tener en cuenta en la comunicación corporativa en página Web supone entender que si dicha comunicación es importante para la organización ésta deberá ser acreedora de un espacio específico, diferenciado del resto de contenidos, y que además dicho tipo de comunicación ocupará un lugar estructuralmente importante en la gestión de contenidos de dicha página. No obstante, es también posible que la comunicación corporativa no sea considerada crucial para las organizaciones, en cuyo caso esta información podría estar tanto subordinada a otros contenidos como, simplemente, no tener presencia en la página Web. 
Los resultados indican la presencia en casi todos los casos (98\% de universidades públicas, y 95,7\% de universidades privadas) de un espacio específico destinado a la comunicación corporativa, y además ésta se encuentra en un lugar estructural preeminente en la página Web (en término medio, el 91,7\% de las organizaciones sitúa el acceso a un clic de distancia, el 1,4\% presenta dicha información en la página de inicio, y el 6,9\% restante a una distancia de dos clicks, sin haberse encontrado diferencias relevantes en función de la titularidad de la universidad), lo que, a priori, hace sugerir que estas organizaciones son conscientes de la importancia que tiene para sus públicos la provisión de información corporativa. Los resultados estratificados pueden apreciarse en la tabla III.

Los títulos que mayoritariamente se dan a esta sección son "conócenos" o "conoce" y el nombre de la universidad, “información”, o las siglas con el nombre de la universidad, a veces acompañada del artículo determinado "la".

Sin embargo, una cuestión es que se disponga de una sección específicamente destinada a albergar información corporativa -que debería estar constituida por la identidad corporativa, tanto a nivel sígnico como conceptual- , y otra bien distinta es la presencia de contenidos específicos en dicha sección, o todavía más, que la totalidad de contenidos corporativos se ubiquen en esta sección. Por ello, se analizó si existían dichos contenidos, y además si los mismos estaban ubicados en dicha sección, o si por el contrario se encuentran ubicados en otras secciones.

\subsection{Información corporativa textual}

Dentro de éstos, se estudia la presencia y ubicación de biografías en la página Web. Los resultados sugieren la escasa presencia de este elemento en las mismas, situándose únicamente en el $21,7 \%$ de las universidades privadas y el 17,6\% de las públicas, aunque cuando tienen presencia se sitúan mayoritariamente dentro de la sección de información corporativa, y marginalmente en otras ubicaciones. Respecto a quiénes son objeto de relato biográfico, destaca la presencia de fundadores o similares, y rectores y vicerrectores, aunque, como ya se ha mencionado, la presencia de este elemento es muy escasa. Los resultados concretos pueden apreciarse en la tabla IV. Por otra parte, como fácilmente puede sospecharse, se ha podido constatar la relación positiva y significativa entre presencia y ubicación de biografías y fotografías de cargos relevantes. Es decir, que cuanto más y mejor aparece las biografías en la página Web, más y mejor situadas aparecen las fotografías de cargos relevantes ${ }^{4}$.

El estadístico Rho de Spearman ofrece un valor de 0,370 y el indicador Tau_b de Kendall señala un valor de 0,362, con un nivel de significación a 0,01 . 
Tendencia distinta puede apreciarse respecto a los informes financieros. Los resultados permiten afirmar que las organizaciones privadas muestran en mucha menor medida esta información que las universidades públicas, tal y como puede apreciarse en la tabla V. Dicha diferencia cobra sentido cuando el objeto específico de las primeras pasa por la generación de beneficio empresarial, por lo que la muestra de interesantes beneficios en su caso podría levantar suspicacias, si bien ha de señalarse que dicha información en todo caso siempre debiera tener presencia en la página Web. En el caso de suponer la presencia de este elemento, suele ubicarse en la sección de información corporativa, siendo en nuestra opinión lo deseable, pues la transparencia también implica facilidad de acceso a la información.

La historia de la institución muestra mayor independencia respecto a la titularidad de la organización, según puede verse también en la tabla V. Su presencia es, en todos casos, frecuente $(86,3 \%$ de universidades públicas, y 91,3\% de universidades privadas), y además su ubicación es la adecuada, ya que se sitúa en la sección de información corporativa. Continuando con esta cuestión, también se analizó la referencia explícita a mitos, al considerarse éstos una formación simbólica de extraordinaria importancia en ese contexto. Si bien suelen tener mayor importancia a nivel interno, son también interesantes cuando en el caso de comunicación externa. Suelen hacer referencia a personas de suma importancia en el desarrollo de la historia organizativa, y usualmente ven incrementadas sus cualidades al extremo de la heroicidad. A nivel interno suponen un referente para el personal. No obstante, los datos obtenidos permiten señalar que la presencia de mitos en el relato de la historia de las universidades es escaso, cifrándose únicamente en el 26,1\% de las universidades públicas y el 13,1\% de las privadas, lo que en nuestra opinión debiera ser mejorado, ya que el coste de manipulación de esta información en principio debiera considerarse bajo, al tratarse de datos de gran estaticidad.

La presencia de valores y misión de la institución fueron también analizados. También constituyen una información sumamente importante, pues sitúan a la universidad en el contexto social y permiten la particularización del discurso dentro de tal contexto. Los datos permiten sugerir una presencia menos frecuente que el caso de la historia (concretamente el $76 \%$ de las universidades públicas y $87 \%$ de las privadas), y una ubicación prácticamente total en la sección de información corporativa, lo cual supone un acierto en tanto que su presencia constituye la declaración de el qué y el cómo de la organización. Los resultados estratificados se muestran en la tabla V. Igualmente, se ha podido constatar una relación positiva y significativa entre presencia y ubicación de la historia de la compañía y la presencia de misión y valores organizativos 5 .

En concreto, los valores arrojados por los estadísticos Rho de Spearman y Tau_b de Kendall son 0,311 y 0,309 respectivamente, ambos significativos a nivel 0,01 . 
El organigrama de estas organizaciones también tiene un reflejo frecuente en sus páginas Web (con presencia en el 96,1\% de las universidades públicas, y el 78,3\% de las privadas), y también muestra una ubicación adecuada (el 84,3\% de las universidades públicas ubican esta información en la sección de información corporativa, y la totalidad de las privadas en la misma sección), según puede apreciarse en la tabla VI.

Por otra parte, la existencia de un calendario de eventos en la página Web es un hecho frecuente en las universidades, concretándose en el 68,6\% de las universidades públicas, y el 65,2\% en el caso de las privadas, según lo dispuesto en la tabla VI. Su ubicación presenta cierta variabilidad, situándose en mayor medida en la página de inicio, y posteriormente en la sala de prensa, siendo marginal su presencia en la sección de información corporativa. Resulta interesante su presencia ya que supone una valiosa fuente de información para los públicos de la universidad, y además para los medios de comunicación, que pudieran con esta información convertirse en eficaces transmisores de discurso para las universidades.

Situación distinta es la planteada por el apartado de Responsabilidad Social Corporativa (RSC), si bien debe entenderse que su objeto de negocio, la educación, podría constituir un bien es sí mismo. La presencia de este elemento en un apartado específico, y por tanto el reconocimiento de la importancia de esta información en su mix de comunicación corporativa, es escaso en el caso de las universidades públicas (tan sólo el $29,4 \%$ mantienen esta característica) y marginal en el caso de las universidades privadas (la presencia de esta información en una sección específica se da únicamente en el 9,1\% de las universidades privadas), tal y como puede verse en la tabla VI. Si bien en el caso de las universidades públicas la educación y la falta de ánimo de lucro constituyen un fin en sí mismo, no ocurre lo mismo con las universidades privadas, que entendemos debieran considerar la presencia de esta información, ya que su ánimo de lucro exige la manifestación de algún tipo de retorno a la sociedad, cuestión que podrían resolver con la publicidad de programas de RSC.

\subsection{Información corporativa icónica}

Mención aparte requieren otros elementos icónicos al margen de la información puramente textual, como son los símbolos, logotipos o fotografías de edificios o instalaciones, que constituyen una fuente de información irrenunciable en la construcción de discurso e identidad desde una perspectiva corporativa. Por ello, también se incluyó en esta investigación el análisis de la presencia y ubicación tanto de logotipos y símbolos como fotografías e ilustraciones de edificios e instalaciones.

Las fotografías son otro recurso interesante a incluir en la página Web. En el caso de nuestro objeto de estudio la presencia de un archivo fotográfico organizado es mayoritaria, algo superior en el caso de las 
universidades privadas, tal y como puede apreciarse en la tabla VII. En dicho archivo cobran especial presencia las fotografías de edificios o instalaciones, con porcentajes de presencia del $87 \%$ en las universidades privadas y del $96 \%$ en el caso de las públicas, lo cual consideramos interesante ya que las fotografías de entorno son también una valiosa fuente de generación de discurso organizativo. Las fotografías de personas relevantes suelen aparecer, cuando esta presencia es un hecho-circunstancia que se produce únicamente en el 41,2\% de las universidades públicas y 40,9\% de las universidades privadas-, vinculadas al organigrama organizativo. Por ello, en todo caso la presencia de estos dos elementos es mayoritaria en la sección de información corporativa, tal y como puede apreciarse en la tabla antes mencionada.

Los datos resultantes de tal análisis, visibles en la tabla VIII, permiten sugerir la presencia muy frecuente (92\% de las universidades públicas y 91,3\% de las privadas) de logotipos y símbolos en las páginas Web de las universidades estudiadas. En general, este hecho se debe a la presencia sistemática de logotipos y símbolos en la cabecera de las páginas pertenecientes a dichas organizaciones. La situación deseable, que sería la presencia de logotipos descargables o la presencia de un manual de identidad visual corporativa, se registra en tan sólo el 18\% de las universidades públicas y el 13\% de las privadas.

\subsection{Información corporativa multimedia}

Sin embargo, otra cuestión es la exposición de contenido mediante audio y vídeo. En lo que respecta al audio, hay que señalar la presencia residual de este elemento en todos los estratos analizados. Además, cuando tiene presencia, la locución se realiza en un único idioma, limitando por tanto el uso de dicho recurso. Por tanto, debemos afirmar que en el caso de los archivos de audio no se aprovecha el potencial de Internet para ofrecer formatos alternativos al texto y la imagen que bien pudieran ser aprovechados por ciertos medios de comunicación, y llegar por tanto a la audiencia que recurre a tales medios para informarse. Los datos pueden apreciarse en la tabla VIII.

La presencia de archivos de vídeo promocionales muestra un comportamiento menos uniforme que el antes descrito, ya que son usados en mayor medida por las universidades privadas $(63,1 \%$ de las universidades privadas lo usan, frente al 41,2\% de las públicas). En el caso de existir tales vídeos en página Web, éstos van sólo parcialmente provistos de descripciones (50\% en universidades privadas y $40 \%$ de públicas), lo que constituye una evidente falta de atención que debería ser corregida. 
Por otra parte, se comprobó que no existía una relación significativa entre presencia y ubicación de audio y vídeos promocionales, que en principio pudiera considerarse ya que respondería al concepto de sensibilidad hacia lo audiovisual en sentido amplio ${ }^{6}$.

Por tanto, consideramos que se desaprovecha la flexibilidad de Internet para la transmisión de discurso organizativo mediante vídeo y audio, lo que entendemos es un error de amplio espectro.

\subsection{Información corporativa y nuevas formas de comunicación en la Web}

La Web 2.0, o Web participativa, supone un interesante conjunto de herramientas sobre la que articular la construcción de discurso corporativo. Sin duda, la articulación de discurso se ve ampliada si se usan estos elementos, muy usados por un público fundamental para estas organizaciones como son los estudiantes, ya que ofrecen un medio de retroalimentación de información potente, y también un medio vehicular de transmisión de contenido muy apto para los estudiantes, ya que tiene por característica fundamental la personalización, pudiendo llegar incluso a la construcción pormenorizada y adaptada de discurso corporativo.

Los datos analizados para nuevas formas de comunicación ofrecen una visión más optimista de estas organizaciones, aunque a nuestro parecer insuficiente, tal y como puede apreciarse en la tabla IX.

En cuanto a la implementación de un sistema de alertas de noticias por móvil -usando las tradicionales conexiones por GPRS al margen de posibles conexiones a Internet en los mismos dispositivos-, los datos muestran su presencia marginal en las universidades. No obstante, considerando que los mismos datos señalan que existe una presencia frecuente de un servicio de sindicación de noticias por RSS en los dos casos, y la proliferación de teléfonos móviles con conexión a Internet, consideramos que el sistema de alertas es un sistema prescindible para las universidades, siempre y cuando se disponga del mencionado sistema de sindicación de noticias por RSS. No obstante, la situación este elemento es variable: se ubica o bien en la sala de prensa, o fuera de la sala de prensa y de la sección de información corporativa, pero no se ubica en ningún caso en la sección de información corporativa, lo que hace sugerir que el uso de este elemento se ciñe más a la difusión de contenido noticioso o de actualidad que a la construcción de discurso corporativo.

6 Es decir, no se pudo validar la idea de que la página Web que contara con vídeos promocionales implicara también la presencia y ubicación, ambos acertados, de archivos de audio. Los valores de las correlaciones fueron de 0,042 y 0,044 para los estadísticos Tau_b de Kendall y Rho de Spearman, no significativos en ninguno de los casos. 
Los blogs son herramientas fundamentales en la Web 2.0. El porcentaje de presencia de este elemento es algo menos del $20 \%$ en las universidades, sean públicas o privadas. Cuando dicho elemento está presente, la situación más frecuente es que se pueda escribir comentarios (85,7\% de los casos analizados en universidades públicas y $50 \%$ de las universidades privadas), pero no se permite ni escribir noticias ni aún menos la publicación directa de las mismas. Es decir, en tanto que se permite el comentario se inicia un diálogo, pero éste no aprovecha la potencialidad que los blogs ofrecen. Además, su ubicación preferente es fuera de las secciones de información corporativa y la sala de prensa, lo que hace sugerir que su uso preferente no está relacionado con la construcción de relato corporativo. Sin embargo, pudo constatarse una relación directa significativa ${ }^{7}$ entre la presencia y ubicación de blogs y servicios de sindicación por RSS, validando por tanto la idea de uso de la Web participativa en sentido amplio.

Los datos analizados en relación a la presencia de las universidades en redes sociales, expuestos en tabla X, permiten señalar la presencia con mayor intensidad en el caso de las universidades privadas (el 59,09\% de ellas tienen presencia en redes sociales, frente al 43,75\% de las universidades públicas). En cualquier caso, y para ambas, la red social preferida es Facebook, seguida de twitter y tuenti, si bien hay que considerar la alta volatilidad de estas herramientas. En cualquier caso, tanto Facebook como Twitter y Tuenti son las redes sociales más usadas por los jóvenes universitarios, por lo que a priori la elección de las redes sociales en qué participar resulta acertada.

Finalmente, y en cuanto a la presencia de las universidades en YouTube, los datos recrean de nuevo la situación antes descrita: la presencia en universidades privadas de este elemento es sensiblemente superior (63,64\% de universidades privadas, frente al 31,25\% de las públicas). Estos datos permiten sugerir que la generación de contenido multimedia, ya sea con presencia en la página Web o en un canal de YouTube es más frecuente en el caso de universidades privadas, aprovechando en mayor medida el potencial narrativo de este formato y el potencial del canal Internet.

Por tanto, debemos señalar que en el caso de estas organizaciones se desaprovecha el potencial comunicativo de estas herramientas, especialmente en el caso de las universidades públicas, constituyendo en nuestra opinión un error ya que dichas formas de comunicación son muy usadas por los jóvenes, que son precisamente uno de los públicos más importantes para las universidades.

En este caso, los valores arrojados fueron de 0,229 y 0242 para los estadísticos Tau_b de Kendall y Rho de Spearman respectivamente, ambos significativos a nivel 0,05. 


\section{Contraste de hipótesis}

La primera hipótesis de partida, inicial, que señala que las páginas Web de las universidades públicas y privadas albergan, en su mayoría, una sección específica destinada a la comunicación corporativa debe validarse. En efecto, y tal como se ha podido comprobar empíricamente, tanto las universidades públicas como las de titularidad privada incorporan en sus páginas una sección específica de información corporativa.

La segunda de ellas indica que las páginas Web de las universidades públicas no contienen en dicha sección, en su mayoría, información concerniente a comunicación corporativa, al menos biografías, informes financieros, historia, valores y misión, organigrama, calendario de eventos, apartado de RSC y logotipos o símbolos, y las universidades privadas sí lo incluyen.

Sorprendentemente, las páginas Web de universidades públicas contienen, al menos en su mayoría, en sus secciones de información corporativa contenidos concernientes a biografías, informes financieros, historia, valores y misión, organigrama, calendario de eventos y logotipos o símbolos. No obstante, no incluyen un apartado específico de responsabilidad social corporativa.

Por otra parte, aunque la mayoría de las páginas Web de las universidades privadas albergan en la sección de información corporativa contenidos relativos a biografías, historia, valores y misión, organigrama, calendario de eventos, apartado de RSC y logotipos o símbolos, la mayoría no contiene información relativa a informes o memorias financieras ni un apartado específico de RSC. En consecuencia, debemos rechazar la hipótesis $\mathrm{H} 2$.

La tercera hipótesis hace referencia a la generación de discurso corporativo usando formatos multimedia, mayoritario en el caso de universidades de titularidad privada. A ese respecto se ha podido constatar que ni las universidades públicas ni las privadas usan este tipo de formatos, por lo que la hipótesis H3 debe ser refutada.

Por último, la cuarta hipótesis señalaba la mayor presencia de herramientas de la Web 2.0 -blogs, servicios de alerta por móvil, sindicación de noticias por RSS, presencia en redes sociales y canales de YouTube- en universidades privadas que en las de titularidad pública. Si bien los datos muestran una presencia muy superior en redes sociales y YouTube para las universidades privadas frente a las públicas, no muestran mayor preponderancia en el caso de servicios de sindicación de noticias por RSS. Es más, en lo relativo a este último elemento, la presencia es mayor en el caso de universidades de titularidad pública. Además, la presencia de blogs es minoritaria y parecida en términos cuantitativos en ambos casos. Finalmente, la pre- 
sencia de un servicio de alertas por móvil es marginal también en ambos casos. Por tanto, la cuarta hipótesis debe ser también rechazada.

\section{Conclusiones y discusión de resultados}

La presencia en Internet y la asignación de un espacio específico para albergar contenidos de tipo corporativo es para las universidades españolas un hecho; por lo que cabe sugerir que dichas organizaciones, sean de titularidad pública o privada, son conscientes de la relevancia que el medio tiene para sus públicos al menos en lo relativo a la difusión de información de este tipo.

En cuanto a los contenidos corporativos de tipo textual, se debe concluir cierta similitud entre universidades públicas y privadas. Todavía más, las universidades públicas se muestran superiores en algún aspecto. Resulta sorprendente en la medida en cómo la diferencia de coste entre unas y otras puede justificar un esfuerzo comunicativo superior. Las universidades privadas deben establecer a nivel corporativo un discurso fuerte que diferencie sensiblemente su oferta de sus competidoras, que sería conveniente que partiera de un riguroso análisis de su identidad corporativa, tanto a nivel sígnico como conceptual.

Así, se debe hablar en primer lugar de la presencia del relato de la historia y los valores y misión de dichas organizaciones. Son elementos de particular importancia ya que contextualizan a la organización dentro de su mercado, situando la particularidad de la misma frente a sus competidores. En este caso, las universidades públicas y privadas cumplen con lo debido y sitúan los contenidos de forma adecuada y coherente, formando un conjunto de ítems, pero con un único inconveniente: deberían incorporarse en mayor medida alusiones a mitos en el relato de la historia, ya que como se dijo anteriormente constituyen referentes simbólicos de gran importancia en la construcción del discurso organizativo. Otra cuestión es la calidad del relato, o la interpretación del mismo por los públicos, aspectos que no son objeto de este trabajo.

Resultaría interesante la presencia en mayor medida de biografías, ya que las mismas también actúan como referentes para el resto de trabajadores, y establecen un modelo vital o conductual a imitar, ya que en alguna medida constituyen símbolos de éxito en tal entorno organizacional.

También sería de interés que las universidades privadas incorporaran en mayor medida elementos fundamentales de transparencia y reputación corporativa tales como informes financieros y elementos de Responsabilidad Social Corporativa, que también son fuente de creación de imagen y por tanto de discurso corporativo. 
El calendario de eventos constituye una información relevante: aporta agilidad al proceso comunicativo, y por tanto consideramos muy conveniente su presencia para los públicos, especialmente los medios de comunicación, que debemos de convertir en eficaces aliados en la transmisión de discurso al resto de públicos.

En cuanto a los contenidos corporativos multimedia, debemos señalar que las universidades, sean públicas o privadas, no explotan adecuadamente estos recursos.

Así, la presencia de logotipos y símbolos organizativos es un hecho en la página de inicio, pero no lo es la presencia de un manual de identidad visual corporativa, que entendemos debe figurar en la página Web, así como otros elementos corporativos.

Todavía más, la existencia de información multimedia (audio y vídeo) de tipo corporativo es cuando menos escasa, y además su presencia en conjunto no ofrece la debida estructuración, lo que hace sugerir que cuando aparecen estos contenidos, lo hacen de forma aislada, sin formar un todo coherente. Dada la plasticidad del soporte Internet y la conveniencia de incorporar información de este tipo en el relato corporativo, parece interesante aumentar su presencia. Más aún cuando en un entorno de competencia tan agresivo como es el de las universidades, en muchos casos el contenido emocional del relato corporativo puede resolverse muy adecuadamente recurriendo a formatos multimedia. Tal circunstancia se da tanto en universidades de titularidad pública como privada.

Finalmente, el uso de la Web 2.0 por parte de las universidades es sólo parcial. Si bien se observa cierto nivel de presencia en redes sociales y YouTube -mayoritaria en el caso de universidades de titularidad privaday servicios de sindicación de noticias por RSS, la ausencia de otros elementos fundamentales como los blogs o los servicios de alerta por móvil resulta patente. Llama la atención que no se usen los blogs, que son canales de uso masivo por parte de estudiantes y por tanto un medio vehicular de transmisión de información corporativa cuando menos muy apto. Además, cuando su improbable presencia es un hecho, su ubicación no es en la sección de información corporativa, lo que hace pensar que su objetivo no es la construcción de relato corporativo. Así, entendemos que la presencia de un blog con propósitos corporativos resultaría un instrumento interesante con la que contar en Internet, más aún considerando la importancia que este canal tiene para los jóvenes universitarios.

Además, a través de herramientas como las redes sociales o YouTube, dada su inherente plasticidad, podrían incorporarse a la oferta comunicativa corporativa en mayor medida, y proponer nuevas formas de promoción corporativa tales como concursos, promociones o sorteos, que son fácilmente gestionables con el uso de estos soportes, aportando originalidad y especificidad al relato corporativo. 
En definitiva, debemos concluir la inadecuada gestión de la comunicación corporativa en este medio, ya que en primer lugar consideramos que se desaprovecha la flexibilidad de soportes que le es inherente, al contener información mayoritariamente textual y prescindir de otras opciones. Además, aunque la presencia de una sección específica en las páginas analizadas es mayoritaria, la gestión de contenidos de la misma no puede calificarse de buena, ya que la misma no contiene toda la información que debiera, y todavía más, no se aprovecha debidamente la flexibilidad del soporte para generar discursos multimedia.

Por todo ello, consideramos que estas organizaciones no aprovechan el potencial que este canal tiene-que combina un generalmente bajo coste de implantación de la información con un elevado margen de explotación estratégica-, y con ello se desperdicia la posibilidad de desarrollar la comunicación corporativa de las universidades en mayor medida.

Las limitaciones más importantes de este estudio se relacionan con el contenido mismo del discurso. Es decir, se analiza qué presencia tienen ciertos contenidos corporativos, pero no se estudia ni el contenido del discurso corporativo, ni los efectos que dicho discurso generan sobre los públicos.

Debemos también aclarar que en este trabajo se estudia únicamente la parte pública de las Web universitarias, excluyendo por tanto la parte privada, tal como la intranet, el campus virtual, etc., que también son fuente fundamental de creación de relato corporativo.

Futuras líneas de investigación podrían incluir el estudio del contenido, o los efectos de dicho contenido sobre el público. Además, podría interesar el estudio de universidades de otros países con un entorno competitivo similar, o incluso el estudio de la comunicación corporativa por otros medios y canales diferentes de Internet. 


\section{Tablas de datos}

Tabla I. Listado de Universidades usadas en el estudio

\begin{tabular}{|lcc|}
\hline Univ. de La Laguna & Univ. Abad Oliba CEU & $\begin{array}{c}\text { Univ. Nacional de Educación a } \\
\text { Distancia (UNED) }\end{array}$ \\
\hline $\begin{array}{l}\text { Univ. de Las Palmas de Gran } \\
\text { Canaria }\end{array}$ & Univ. Abierta de Cataluña (UOC) & Univ. Politécnica de Madrid \\
\hline Univ. de Cantabria & Univ. Internacional de Cataluña & Univ. Rey Juan Carlos \\
\hline Univ. Internacional Menéndez Pelayo & Univ. Ramon Llull & Univ. a Distancia de Madrid \\
\hline Univ. de Castilla-La Mancha & Univ. de Vic & Univ. Alfonso X el Sabio \\
\hline Univ. de Burgos & Univ. de Alicante & Univ. Antonio de Nebrija \\
\hline Univ. de León & Univ. Miguel Hernández de Elche & Univ. Camilo José Cela \\
\hline Univ. de Salamanca & Univ. Jaime I & Univ. CEU San Pablo \\
\hline Univ. de Valladolid & Univ. de Valencia & Univ. Europea de Madrid \\
\hline Univ. Católica de Ávila & Univ. Politécnica de Valencia & Univ. Francisco de Vitoria \\
\hline Univ. Internacional de Andalucía & Univ. Católica San Vicente Mártir & Univ. Pontificia Comillas \\
\hline Univ. Pontificia de Salamanca & Univ. CEU Cardenal Herrera & Univ. de Murcia \\
\hline IE Universidad & Univ. de Extremadura & Univ. Politécnica de Cartagena \\
\hline Univ. Europea Miguel de Cervantes & Univ. de La Coruña & Univ. Católica San Antonio \\
\hline Univ. de Barcelona & Univ. de Santiago de Compostela & Univ. Pública de Navarra \\
\hline Univ. Politécnica de Cataluña & Univ. de Vigo & Univ. de Navarra \\
\hline Univ. Pompeu Fabra & Univ. de La Rioja & Univ. del País Vasco \\
\hline Univ. Autónoma de Barcelona & Univ. Autónoma de Madrid & Univ. de Deusto \\
\hline Univ. de Gerona & Univ. Carlos III de Madrid & Univ. de Mondragón \\
\hline Univ. de Lérida & Univ. Complutense de Madrid & \\
\hline Univ. Rovira i Virgili & Univ. de Alcalá & \\
\hline
\end{tabular}


Tabla II. Idiomas disponibles en Página Web

\begin{tabular}{|lccccc|}
\hline Sólo en Español & $\begin{array}{c}\text { Español e } \\
\text { Inglés }\end{array}$ & $\begin{array}{c}\text { Español y } \\
\text { otra distinta } \\
\text { de inglés }\end{array}$ & $\begin{array}{c}\text { Más de dos } \\
\text { idiomas }\end{array}$ & Total \\
\hline Pública & $7,84 \%$ & $41,18 \%$ & $1,96 \%$ & $49,02 \%$ & $100 \%$ \\
\hline Privada & $14,29 \%$ & $38,10 \%$ & - & $47,62 \%$ & $100 \%$ \\
\hline Total & $9,72 \%$ & $40,28 \%$ & $1,39 \%$ & $48,61 \%$ & $100 \%$ \\
\hline
\end{tabular}

Tabla III. Número de clicks necesarios para acceder a la información corporativa

\begin{tabular}{|lcccc|}
\hline & 0 & 1 & 2 & Total \\
\hline Pública & $2 \%$ & $88 \%$ & $10 \%$ & $100 \%$ \\
\hline Privada & - & $100 \%$ & - & $100 \%$ \\
\hline Total & $1,39 \%$ & $91,67 \%$ & $6,94 \%$ & $100 \%$ \\
\hline
\end{tabular}

Tabla IV. Qué biografías hay en la página

\begin{tabular}{|lccccc|}
\hline & $\begin{array}{c}\text { Sólo la del fundador } / \\
\text { rector / gerente }\end{array}$ & Fundador y rector & $\begin{array}{c}\text { Fundador, rector, } \\
\text { vicerrectores }\end{array}$ & Otras & Total \\
\hline Pública & $22,22 \%$ & - & $66,67 \%$ & $11,11 \%$ & $100 \%$ \\
\hline Privada & - & $40,00 \%$ & $60,00 \%$ & - & $100 \%$ \\
\hline Total & $14,29 \%$ & $14,29 \%$ & $64,29 \%$ & $7,14 \%$ & $100 \%$ \\
\hline
\end{tabular}


Tabla V. Ítems de información corporativa (I)

\begin{tabular}{|llccccc|}
\hline & & $\begin{array}{c}\text { Si, en la sección } \\
\text { de información } \\
\text { corporativa }\end{array}$ & $\begin{array}{c}\text { Si, en la sala } \\
\text { de prensa }\end{array}$ & $\begin{array}{c}\text { Si, fuera } \\
\text { de ambas }\end{array}$ & No & Total \\
\hline Informes financieros/ & Pública & $51 \%$ & $2 \%$ & $7,8 \%$ & $39,2 \%$ & $100 \%$ \\
memorias actividad & Privada & $21,7 \%$ & $4,4 \%$ & - & $73,9 \%$ & $100 \%$ \\
\hline Historia de la & Pública & $86,3 \%$ & - & - & $13,7 \%$ & $100 \%$ \\
organización & Privada & $91,3 \%$ & - & - & $8,7 \%$ & $100 \%$ \\
\hline Valores y misión & Pública & $74 \%$ & - & $2 \%$ & $24 \%$ & $100 \%$ \\
& Privada & $87 \%$ & - & - & $13 \%$ & $100 \%$ \\
\hline
\end{tabular}

Tabla VI. Ítems de información corporativa (II)

\begin{tabular}{|llccccc|}
\hline & & $\begin{array}{c}\text { Si, en la sección } \\
\text { de información } \\
\text { corporativa }\end{array}$ & $\begin{array}{c}\text { Si, en la sala } \\
\text { de prensa }\end{array}$ & $\begin{array}{c}\text { Si, fuera } \\
\text { de ambas }\end{array}$ & No & Total \\
\hline Organigrama & Pública & $84,3 \%$ & - & $11,8 \%$ & $3,9 \%$ & $100 \%$ \\
& Privada & $78,3 \%$ & - & - & $21,7 \%$ & $100 \%$ \\
\hline Calendario de eventos & Pública & $3,9 \%$ & $27,5 \%$ & $37,2 \%$ & $31,4 \%$ & $100 \%$ \\
& Privada & - & $21,7 \%$ & $43,5 \%$ & $34,8 \%$ & $100 \%$ \\
\hline Apartado RSC & Pública & $16,7 \%$ & - & $12,7 \%$ & $70,6 \%$ & $100 \%$ \\
& Privada & - & - & $9,1 \%$ & $90,9 \%$ & $100 \%$ \\
\hline
\end{tabular}


Tabla VII. Ítems de información corporativa (III)

\begin{tabular}{|llccccc|}
\hline & & $\begin{array}{c}\text { Si, en la sección } \\
\text { de información } \\
\text { corporativa }\end{array}$ & $\begin{array}{c}\text { Si, en la sala } \\
\text { de prensa }\end{array}$ & $\begin{array}{c}\text { Si, fuera } \\
\text { de ambas }\end{array}$ & No & Total \\
\hline Archivo fotográfico & Pública & $54,9 \%$ & $7,8 \%$ & $19,6 \%$ & $17,7 \%$ & $100 \%$ \\
& Privada & $47,8 \%$ & $21,7 \%$ & $21,7 \%$ & $8,8 \%$ & $100 \%$ \\
\hline Fotografías cargos & Pública & $36,3 \%$ & & $4,9 \%$ & $58,8 \%$ & $100 \%$ \\
relevantes & Privada & $36,4 \%$ & - & $4,5 \%$ & $59,1 \%$ & $100 \%$ \\
\hline Fotografías edificios & Pública & $66 \%$ & $4 \%$ & $26 \%$ & $4 \%$ & $100 \%$ \\
e instalaciones & Privada & $60,9 \%$ & $13 \%$ & $13 \%$ & $13,1 \%$ & $100 \%$ \\
\hline
\end{tabular}

Tabla VIII. Ítems de información corporativa (IV)

\begin{tabular}{|llccccc|}
\hline & & $\begin{array}{c}\text { Si, en la sección } \\
\text { de información } \\
\text { corporativa }\end{array}$ & $\begin{array}{c}\text { Si, en la sala } \\
\text { de prensa }\end{array}$ & $\begin{array}{c}\text { Si, fuera } \\
\text { de ambas }\end{array}$ & No & Total \\
\hline Logos y símbolos & Pública & $18 \%$ & $2 \%$ & $72 \%$ & $8 \%$ & $100 \%$ \\
& Privada & $13 \%$ & - & $78,3 \%$ & $8,7 \%$ & $100 \%$ \\
\hline Vídeos promocionales & Pública & $21,6 \%$ & - & $18,6 \%$ & $59,8 \%$ & $100 \%$ \\
& Privada & - & - & $63,1 \%$ & $36,9 \%$ & $100 \%$ \\
\hline Audio & Pública & - & $3,9 \%$ & $2 \%$ & $94,1 \%$ & $100 \%$ \\
& Privada & - & - & $4,3 \%$ & $95,7 \%$ & $100 \%$ \\
\hline
\end{tabular}


Tabla IX. Nuevas formas de comunicación. (I)

\begin{tabular}{|lccccc|}
\hline & & $\begin{array}{c}\text { Si, en la Sala } \\
\text { de Prensa }\end{array}$ & $\begin{array}{c}\text { Si, fuera de la Salade Prensa } \\
\text { y de la info corporativa }\end{array}$ & No & Total \\
\hline \multirow{2}{*}{ Existe un blog en la Web? } & Pública & & $18,00 \%$ & $82,00 \%$ & $100 \%$ \\
& Privada & $4,55 \%$ & $18,18 \%$ & $77,27 \%$ & $100 \%$ \\
& Total & $1,39 \%$ & $18,06 \%$ & $80,56 \%$ & $100 \%$ \\
\hline Servicio de alertas por & Pública & $2,04 \%$ & & $97,96 \%$ & $100 \%$ \\
móvil & Privada & & & $100,00 \%$ & $100 \%$ \\
& Total & $1,39 \%$ & $36,00 \%$ & $26,00 \%$ & $100 \%$ \\
\hline Servicio sindicación & Pública & $38,00 \%$ & $30,43 \%$ & $30,43 \%$ & $100 \%$ \\
noticias por RSS & Privada & $39,13 \%$ & $34,25 \%$ & $27,40 \%$ & $100 \%$ \\
\hline
\end{tabular}

Tabla X. Nuevas formas de comunicación. (II)

\begin{tabular}{|lcccc|}
\hline & & No & Si & Total \\
\hline ¿Se pueden mandar noticias, etc a las redes sociales? & Pública & $56,25 \%$ & $43,75 \%$ & $100 \%$ \\
& Privada & $40,91 \%$ & $59,09 \%$ & $100 \%$ \\
& Total & $51,43 \%$ & $48,57 \%$ & $100 \%$ \\
\hline ¿Tienen un canal de YouTube? & Pública & $68,75 \%$ & $31,25 \%$ & $100 \%$ \\
& Privada & $36,36 \%$ & $63,64 \%$ & $100 \%$ \\
& Total & $58,57 \%$ & $41,43 \%$ & $100 \%$ \\
\hline
\end{tabular}

\section{Anexo: Plantilla de análisis}

1. Nombre de la Universidad

2. Pública / Privada

3. ¿Tiene Web?

NO / SI 
4. ¿En qué idiomas está disponible?

1. Sólo en Español

2. Español e Inglés

3. Español y otra distinta del inglés

4. Más de dos idiomas

\section{INFORMACIÓN CORPORATIVA}

5. ¿Existe una sección específica con información corporativa?

6. Nombre de la sección

7. Número de clicks necesarios para acceder a la sección

8. ¿ ¿Hay biografías en la Web?

1. Si, en la sección de Info. Corporativa

2. Si, en la Sala de prensa
3. Si, fuera de la SP y de la Inf. Corporativa

4. No

9. ¿Qué biografías hay?

1. Sólo la del fundador / rector / gerente

2. Fundador y rector / gerente

3. Fundador, Rector, Vicerrectores

4. Fundador, Rector, Vicerrectores y Profesores

5. Otras

10. ¿Hay informes financieros o memorias de actividad (ej, cuentas anuales) en la Web?

1. Si, en la sección de Info. Corporativa

3. Si, fuera de la SP y de la Inf. Corporativa

2. Si, en la Sala de prensa

4. No

11. ¿Consta la historia de la compañía en la Web?

1. Si, en la sección de Info. Corporativa

3. Si, fuera de la SP y de la Inf. Corporativa

2. Si, en la Sala de prensa

4. No 
12. ¿Hay referencias explícitas a mitos (ej. fundadores) en la historia de la compañía?

13. ¿Constan los valores y la filosofía de la institución?
1. Si, en la sección de Info. Corporativa
3. Si, fuera de la SP y de la Inf. Corporativa
2. Si, en la Sala de prensa
4. No

14. ¿Consta el organigrama de la organización?
1. Si, en la sección de Info. Corporativa
3. Si, fuera de la SP y de la Inf Corporativa
2. Si, en la Sala de prensa
4. No

15. ¿Existe un apartado específico de Responsabilidad Social Corporativa (RSC)?
1. Si, en la sección de Info. Corporativa
3. Si, fuera de la SP y de la Inf. Corporativa
2. Si, en la Sala de prensa
4. No

\section{MULTIMEDIA.- FOTOGRAFÍA}

16. ¿Hay archivo fotográfico en la Web?

1. Si, en la sección de Info. Corporativa

3. Si, fuera de la SP y de la Inf. Corporativa

2. Si, en la Sala de prensa

4. No

17. ¿Tiene el archivo fotográfico reseñas o comentarios sobre las fotos? NO / SI

18. ¿Hay fotografías de los actos académicos realizados?

1. Si, en la Sala de Prensa

2. Si, en la sección de Info. Corporativa
3. Si, fuera de la SP y de la Inf Corporativa

4. No

19. ¿Hay fotos de los cargos relevantes y/o profesores?

1. Si, en la sección de Info. Corporativa

3. Si, fuera de la SP y de la Inf. Corporativa

2. Si, en la Sala de prensa

4. No

20. ¿ ¿Hay fotos de los edificios, ya sean externas al mismo o de las instalaciones?

1. Si, en la sección de Info. Corporativa

3. Si, fuera de la SP y de la Inf. Corporativa

2. Si, en la Sala de prensa

4. No 
21. ¿Tiene la Web logos de la institución?

1. Si, en la sección de Info. Corporativa

2. Si, en la Sala de prensa

22. ¿Se pueden descargar las fotos de eventos, etc,...?
3. Si, fuera de la SP y de la Inf. Corporativa

4. No

$\mathrm{NO} / \mathrm{SI}$

\section{MULTIMEDIA.- VIDEOS / AUDIO}

23. ¿ ¿Hay vídeos con actos realizados en la Web?

1. Si, en la Sala de prensa

2. Si, en la sección de Info. Corporativa

3. Si, fuera de la SP y de la Inf. Corporativa

4. No

24. ¿ ¿Hay vídeos promocionales en la Web?

1. Si, en la sección de Info. Corporativa

3. Si, fuera de la SP y de la Inf. Corporativa

2. Si, en la Sala de prensa

4. No

25. ¿ ¿Hay descripciones escritas sobre los vídeos?

$\mathrm{NO} / \mathrm{SI}$

26. ¿ ¿Hay archivos de audio en la Web?

1. Si, en la sección de Info. Corporativa

3. Si, fuera de la SP y de la Inf. Corporativa

2. Si, en la Sala de prensa

4. No

27. ¿En cuántos idiomas se ofrecen los archivos de audio?

\section{DATOS DE CONTACTO EN WEB}

28. ¿Hay algún número telefónico de contacto?

1. Si, en la página de inicio

2. Si, en la sección de Info. Corporativa

4. Si, fuera de la SP y de la Inf. Corporativa

3. Si, en la Sala de prensa

5. No

29. ¿Hay algún email de contacto?

1. Si, en la página de inicio

2. Si, en la sección de Info. Corporativa

4. Si, fuera de la SP y de la Inf. Corporativa

$140\left|n^{\circ} 13\right|$ doxa.comunicación 

3. Si, en la Sala de prensa
5. No

30. ¿Hay un formulario para escribir comentarios o sugerencias en la Web?
1. Si, en la sección de Info. Corporativa
3. Si, fuera de la SP y de la Inf. Corporativa
2. Si, en la Sala de prensa
4. No

\section{NUEVAS TECNOLOGÍAS / FORMAS DE COMUNICACIÓN}

31. ¿ ¿Hay chats o similares para mantener entrevistas online?

1. Si, en la Sala de prensa

2. Si, en la sección de Info. Corporativa
3. Si, fuera de la SP y de la Inf. Corporativa 4. No

32. ¿Existe la posibilidad de suscribirse a newsletters o boletines periódicos?

1. Si, en la sección de Info. Corporativa

3. Si, fuera de la SP y de la Inf. Corporativa

2. Si, en la Sala de prensa

4. No

33. ¿Es necesario suscribirse para verlo o recibirlo? NO / SI

34. ¿Qué periodicidad tiene?
1. Diario
3. Mensual
2. Semanal
4. Con mayor periodicidad

35. ¿Existe la posibilidad de suscribirse a un servicio de alertas de noticias por móvil?

1. Si, en la Sala de prensa

2. Si, en la sección de Info. Corporativa

36. ¿Existe un blog?

1. Si, en la Sala de prensa

2. Si, en la sección de Info. Corporativa

37. ¿Se puede comentar el contenido del blog?

38. $\quad$ iSe puede escribir noticias en el blog?
3. Si, fuera de la SP y de la Inf. Corporativa

4. No

3. Si, fuera de la SP y de la Inf. Corporativa

4. No

$\mathrm{NO} / \mathrm{SI}$

$\mathrm{NO} / \mathrm{SI}$ 
39. ¿Existe la posibilidad de suscribirse a un servicio de noticias por RSS?

1. Si, en la Sala de prensa

2. Si, en la sección de Info. Corporativa
3. Si, fuera de la SP y de la Inf. Corporativa

4. No

40. ¿ ¿Se puede mandar la información de las noticias a redes sociales?

NO / SI

41. ¿En qué redes sociales participan?

1. Facebook

3. Twitter

5. Ninguna
2. tuenti

4. mySpace

42. ¿ ¿Hay un canal de YouTube?

$\mathrm{NO} / \mathrm{SI}$

43. ¿Existe un buscador de contenido general en la Web?

$\mathrm{NO} / \mathrm{SI}$

\section{Referencias bibliográficas}

Aguadero, F. (2010): “La responsabilidad social corporativa como punto de encuentro”, REDMARKA, 5. pp. 53-70

Aguillo, I. F.; Granadino, B. (2006): “Indicadores Web para medir la presencia de las universidades en la Red”, en Roca, G. (coord.): La presencia de las universidades en la Red Revista de Universidad y Sociedad del Conocimiento (RUSC), 3, (1). p. 73.

Capriotti, P. (2005): Planificación estratégica de la imagen corporativa. Barcelona: Ariel Comunicación.

Castañeda, G., Ruiz, M., Viloria, O., Castañeda, R. y Quevedo, Y. (2007): “El Rol de las Universidades en el Contexto de la Responsabilidad Social Empresarial”, Negotium: Revista de Ciencias Gerenciales, 8. pp. 100-132

Cruz Álvarez, J.G., Rodríguez, M. P. y Abreu, J. L. (2010): “Gestión de Responsabilidad Social: Análisis Comparativo de Enfoques, Tendencias e Indicadores”, International Journal of Good Conscience, 5 (2), pp. 100-118

Estudio General de Medios (2009): Estudio de la audiencia de Internet. Octubre / Noviembre 2009. [http://www.aimc.es].

Freire, J. (2007): “Los retos y oportunidades de la Web 2.0 para las universidades”, en Jiménez Cano, R y Polo, F. (eds.): $L a$ Gran Guía de los Blogs 2008. Barcelona: Colección Planta29, El Cobre Ediciones, pp. 82-90.

Fumero, A., Roca, G. y Sáez Vacas, F. (2007): Web 2.0. Madrid: Fundación Orange.

García, J. C., Iturrioz, J., Mateu, J. L. y Palomo, R. (2011): “La percepción sobre la responsabilidad social en las sociedades cooperativas de trabajo asociado y las sociedades laborales: un análisis en el ámbito de la ciudad de Madrid”, Revesco, 104, pp.102-124 
Gil Leiva y Moya Martínez (2001): “Evaluación de websites de Universidades Españolas”. Actas InterMedia 2001: Internacional Workshop on Multimedia Applications.

Gordon, J. y Berhow, S. (2009): "University websites and dialogic features for building relationships with potential students”, Public Relations Review, 35 (2), pp. 150-152.

Herranz de la Casa, J. M. (2010): “Comunicar la economía social: cómo difundir otra forma de emprender”, Icade. Revista cuatrimestral de las Facultades de Derecho y Ciencias Económicas y Empresariales, 80, pp. 303-318.

Martínez Grás, R. (1998): “Comunicación interactiva. Internet y la gestión de la comunicación en la universidad”. Seminario Complutense de Telecomunicaciones e Información. Madrid.

Martínez Méndez, J. (2008): “Ubicuidad y usabilidad de los portales Web de las universidades españolas”, Scire, 14 (2), pp. 87-106.

Pinto Molina, M. et al (2004): "Análisis Cualitativo de la visibilidad de la investigación de las universidades españolas a través de sus páginas Web”, Revista Española de Documentación Científica, 27, (3), pp. 345-370.

Raymond, D. (2006): “Temas y dinámica de publicación de las Web de un conjunto de organizaciones. Estudio panorámico y longitudinal del contenido y la página de inicio de los sitios Web de las universidades francesas". Conferencia IADIS Ibero-Americana WWW/Internet 2006, pp. 414-419.

Sanz de la Tajada, L. A. (1996): Auditoría de la imagen de la empresa. Métodos y técnicas de estudio de la imagen. Madrid: Síntesis.

Tapia, A., Gómez, B., Herranz, J.M. y Matellanes, M (2009): "La gestión de la comunicación de las organizaciones de economía social a través de sus portales Web”. Actas de XII Jornadas de Investigadores de Economía Social. Murcia, 2009.

Térmens Graells, M., Ribera Turró, M. y Sulé Duesa, A. (2003): "Nivel de accesibilidad de las sedes Web de las universidades españolas”, Revista Española de Documentación Científica, 26 (1), pp. 21-39.

Thelwall, M. y Aguillo, I. (2003): “La salud de las Web universitarias españolas”, Revista Española de Documentación Científica, 26 (3), pp. 291-305.

Van Riel, C. (2000): Comunicación corporativa. Madrid: Ed. Prentice Hall Pearson Educación.

Villafañe, J. (1993): Imagen positiva. Gestión estratégica de la imagen de las empresas. Madrid: Pirámide. 